\title{
Pulmonary Micronodules Less Than 5 mm Detected At Presentation In Patients With Osteosarcoma Do Not Effect 5 Year Overall Survival
}

\author{
Reid Davison ${ }^{1}$, Fadi Hamati ${ }^{1}$, and Paul Kent ${ }^{2}$ \\ ${ }^{1}$ Rush Medical College of Rush University \\ ${ }^{2}$ Rush University Medical Center
}

October 5, 2020

\begin{abstract}
Within the last 25 years, high-resolution CT scans have revealed pulmonary micronodules (nodules $<10$ mm) not previously seen. For osteosarcoma (OST), staging criteria, prognosis estimates, and surgical recommendations have not yet changed to reflect this reality, however, the frequent identification of micronodules on presentation leaves clinicians in a difficult position regarding the need to biopsy, resect, or follow the lesions and whether to consider the patient metastatic or non-metastatic. We retrospectively collected data on all newly diagnosed OST patients, age less than 50, treated at Rush University Hospital over 25 years without pulmonary nodules $>10 \mathrm{~mm}$ to study the relationship between size and location of micronodules discovered at the time of diagnosis and its relationship to 5 year overall survival. Kaplan-Meier curves comparing 5 year overall survival of patients with their largest nodules on presentation at each size interval showed there was no difference in 5 year overall survival in patients with any size nodule $<5 \mathrm{~mm}$ compared to patients with no nodules. In addition, our study showed a survival advantage for those who presented with 0 or 1 nodule (90\%) compared to [?] 2 nodules (53\%). Additionally, patients who develop nodules after presentation but within a year of diagnosis with any number of new micronodules had a lower 5 year overall survival (93\% vs 63\%). Our data suggest surgery may not be necessary for singular nodules $<5$ mm identified on presentation, and that these patients behave more like "localized" patients than metastatic patients.
\end{abstract}

Pulmonary Micronodules Less Than $5 \mathrm{~mm}$ Detected At Presentation In Patients With Osteosarcoma Do Not Effect 5 Year Overall Survival

Davison, Reid MSE ${ }^{1}$; Hamati, Fadi ${ }^{1}$; Kent, Paul MD ${ }^{1,2}$

${ }^{1}$ Rush University School of Medicine

${ }^{2}$ Pediatric Oncology, Rush University Medical Center

Corresponding author:

Reid Davison

1119 S Loomis St, Apartment 201

Chicago IL, 60607

(248)-622-0988

Reid_m_davison@rush.edu

Word Count: 
Abstract: 247

Main text: 3380

Number of Tables: 1, Figures: 5, Supporting Information files: 0

Short running title: Lung Micronodules in Patients with Osteosarcoma

Keywords: Osteosarcoma, sarcoma, neoplasm metastasis, solitary pulmonary nodule, survival, prognosis

Abbreviations:

\begin{tabular}{ll}
\hline Abbreviation & Term \\
\hline OST & Osteosarcoma \\
OS & Overall survival \\
COG & Children's Oncology Group \\
NCCN & National Comprehensive Cancer Network \\
IRB & Institutional Review Board \\
\hline
\end{tabular}

1. Davison, Reid, Hamadi, Fadi, Kent, Paul, Fewer Than Two Pulmonary Nodules Identified On Presentation In Patients With Osteosarcoma Have No Effect On 5 Year Overall Survival, Connective Tissue Oncology Society, November 2020.

2. Davison, Reid, Hamadi, Fadi, Kent, Paul, Pulmonary Micronodules Less Than $5 \mathrm{~mm}$ Detected On CT At Presentation In Patients With Osteosarcoma Do Not Effect 5 Year Overall Survival, Connective Tissue Oncology Society, November 2020.

\section{ABSTRACT}

\section{Background}

Within the last 25 years, high-resolution CT scans have revealed pulmonary micronodules (nodules $<10$ $\mathrm{mm}$ ) not previously seen. For osteosarcoma (OST), staging criteria, prognosis estimates, and surgical recommendations have not yet changed to reflect this reality, however, the frequent identification of micronodules on presentation leaves clinicians in a difficult position regarding the need to biopsy, resect, or follow the lesions and whether to consider the patient metastatic or non-metastatic.

\section{Procedure}

We retrospectively collected data on all newly diagnosed OST patients, age less than 50, treated at Rush University Hospital over 25 years without pulmonary nodules $>10 \mathrm{~mm}$ to study the relationship between size and location of micronodules discovered at the time of diagnosis and its relationship to 5 year overall survival.

\section{Results}

Kaplan-Meier curves comparing 5 year overall survival of patients with their largest nodules on presentation at each size interval showed there was no difference in 5 year overall survival in patients with any size nodule $<5 \mathrm{~mm}$ compared to patients with no nodules. In addition, our study showed a survival advantage for those who presented with 0 or 1 nodule (90\%) compared to [?] 2 nodules (53\%). Additionally, patients who develop nodules after presentation but within a year of diagnosis with any number of new micronodules had a lower 5 year overall survival (93\% vs $63 \%$ ).

\section{Conclusions}

Our data suggest surgery may not be necessary for singular nodules $<5 \mathrm{~mm}$ identified on presentation, and that these patients behave more like "localized" patients than metastatic patients.

\section{Introduction}


Osteosarcoma (OST) is one of the most common non-hematopoietic bone sarcomas with an annual incidence of 800 to 900 new cases in the United Stated, with about half of these cases involving children and teens, however people of any age can be affected ${ }^{1-4}$. The 5 -year overall survival rate of patients with OST ranges from $28 \%$ to $70 \% .^{5,6}$ This variation has been attributed to several factors including metastasis at diagnosis, age, tumor location, tumor size, and percent necrosis at primary surgery. The biggest improvements are from adjuvant chemotherapy for OST patients classified as "localized" at diagnosis. Because the lungs are the most common site for metastases, all patients with biopsy positive OST receive an initial lung CT. However, it has not been well established how the discovery of lung micronodules at the time of diagnosis effects the 5 -year overall survival or the management of patients with OST, since the dichotomy between "localized" and "metastatic" has become blurred. Since 1996 the Children's Oncology Group (COG) and the National Comprehensive Cancer Network (NCCN) have had an inconsistent and unvalidated definition of "pulmonary metastasis" for subcentimeter lesions based on initial CT imaging for OST. ${ }^{7}$ In patients newly diagnosed with OST, the dichotomous classification of the disease as "localized" or "metastatic" has implications with respect to surgical intervention, clinical trial participation, and prognosis.

The widespread adoption of high resolution CT scans in the last 25 years has revealed pulmonary micronodules (defined as nodules $<10 \mathrm{~mm}$ ) not previously appreciated ${ }^{8}$ and even experienced radiologist have not been in agreement reading micronodules. ${ }^{9}$ This category of nodules is important as many consider lung nodules $<10 \mathrm{~mm}$ as 'non-measurable' and cannot be used as 'target lesions". ${ }^{10}$ These lesions may increase, decrease, or disappear without resolving the question of their etiology, which may include transient atelectasis, recent infection, or simply different degrees of sensitivity by the CT scanner or the radiologist, ${ }^{10}$ in addition to metastatic disease. However the frequent identification of nodules on presentation leaves clinicians in a difficult position regarding the need to biopsy, resect, or follow the lesions, and whether to consider the patient metastatic or non-metastatic for purposes of study enrollment and prognosis counselling. ${ }^{10}$ Osteosarcoma was selected as our population of choice because surgery or biopsy, and not radiation or chemotherapy, as in other sarcomas, is seen as the only curative intervention for detectable lung lesions. In fact, open bilateral surgical thoracic exploration is still recommended in many protocols. The assumed, but unproven assertion is that surgical resection of tiny lung nodules benefits the patient.

Staging criteria, prognosis estimates, and surgical recommendations have not yet changed to reflect the increase in incidence of detecting lung nodules. The "New Response Evaluation Criteria in Solid Tumours: Revised RECIST guideline (version 1.1)" considers lung nodules $<10 \mathrm{~mm}$ as 'non-measurable,' and many clinical trials require RECIST criteria, ie, lesions $>10 \mathrm{~mm}$ for enrollment. ${ }^{10}$ At the COG Annual Meeting in 2008, the COG identified "small pulmonary nodules" as an area requiring additional research; to date, however, the literature remains scant. Additionally, the relationship between percent necrosis of the primary tumor after 2 rounds of chemotherapy, which is an important prognostic indicator for patients with OST, ${ }^{11-14}$ and lung nodule management has yet to be examined.

The few studies that address this issue do not include all patients under the age of 50 years, do not limit it to initial CT findings (which is important because development of new lesions after initial presentation is associated with progressive disease), do not limit it to osteosarcoma (an important consideration since surgery is seen as necessary for curative intent in OST, unlike many other sarcomas), nor studied the effect of surgery versus observation of micronodules on overall survival. ${ }^{15,16}$ For example, Kusam et al, retrospectively studied 27 OST and 8 Ewings sarcoma patients, less than 25 years of age, who had biopsies of lung nodules presenting at any time, and found that nodules $>5 \mathrm{~mm}$ predicted malignancy, and up to $1 / 3$ of those $<$ $5 \mathrm{~mm}$ when found in connection to large nodules $(>5 \mathrm{~mm})$ were malignant but only two patients withonly $<5 \mathrm{~mm}$ nodules found to be malignant after biopsy. ${ }^{17}$ We do not know if these micronodules were present at diagnosis, therefore affecting management, or appeared later, nor if the surgery effected survival. Ghosh, et al, retrospectively studied 104 mostly adult OST patients with "indeterminant nodules" (defined as radiologist unable to call them "benign," or "metastatic," and non-calcified $<10 \mathrm{~mm}$ ), found survival for "indeterminant" nodules similar to those classified as "without metastasis," 18 but we do not know who had surgery and which micronodules were not counted because they were initially read as "benign" or "malignant" or "calcified." 
We sought to address some of these shortcoming using our own extensive database of over 500 patients with osteosarcoma. Our institution has maintained a combined adult and pediatric oncology sarcoma program for 40 years, with a longstanding combined weekly sarcoma tumor board, a tradition of treating all OST patients under 50 uniformly according to standard COG (or current pediatric) protocols, and a large number of patients who did not get surgery for micronodules.

\section{Methods}

Under an IRB approved protocol (IRB number 16071805), we retrospectively collected data on all newly diagnosed OST patients, age less than 50, treated at Rush University Hospital over 25 years from 1995-2020 who had an initial CT chest within two months of diagnosis. Subjects were excluded if they had a history of other prior cancer treatment, any lung nodule $>10 \mathrm{~mm}$, other metastatic disease, or had surgery on a lobe of the lung involved with any nodule on presentation. Diagnosis date was defined as the date of biopsy proven OST. All patients underwent similar treatment protocols following Children's Oncology Group or National Comprehensive Cancer Network's standard of care, which entailed the surgical excision of the primary tumor with adjuvant and neoadjuvant chemotherapeutic treatment, consisting of Methotrexate, cis-platinum, and doxorubicin.

Patient demographics (sex, age at diagnosis, primary tumor location, type of primary resection surgery, percent necrosis, and dates last known alive and dates of death were collected by reviewing electronic medical records and patient charts. Initial CT scan reports within the first month of diagnosis were identified and reviewed for number of nodules, diameters of the nodules, as well as location and laterality of the nodules. Micronodules were identified by reviewers if they were under $10 \mathrm{~mm}$ in size and not explicitly labeled as benign by the reporting radiologist. Huvos scores were calculated based on previously defined criteria for categorizing percent necrosis of a patients' primary tumor after 2 rounds of chemotherapy. ${ }^{19}$

Over the 25-year inclusion period, corresponding to the era of consistent high resolution CT scanning, patients who fit these criteria were divided into cohorts based on the number of nodules on the initial CT scan, the largest nodules on presentation, the number of lung lobes involved, and the timing of nodule occurrence within the first year. In addition an "additive micronodule cohorts," based on RECIST 1.1 for Solid tumors ${ }^{10}$ "sum of target lesions at baseline" paradigm, were constructed, where, for example, a patient with $3 \mathrm{~mm}$ and $4 \mathrm{~mm}$ nodules would be represented as $7 \mathrm{~mm}$. Kaplan-Meier survival curves for years of survival for these groups were also constructed and compared between the cohorts. Data was analyzed using R-Studio version (RStudio: Integrated Development for R. RStudio, PBC, Boston, MA), and an alpha value of 0.05 was considered significant. Tarone-Ware tests analyses were conducted to compare the 5 -year survival between various patient cohorts, and T-tests, chi squared tests, and 1- and 2-way ANOVA analyses were conducted to compare patient characteristics between groups.

\section{Results}

Over 25 years, there were 97 patients (mean age $=20$, range $=6-49,46 \mathrm{~F}, 51 \mathrm{M}$ ) that fit our inclusion and exclusion criteria, with $44(45 \%)$ patients having nodules on presentation and 53 did not. Of the 78 patients who we had conformation at 5 years if they were alive or dead, 61 were still alive (78\%). Figure 1 is a Kaplan Meier curve comparing the 5-year overall survival (OS) of patients with no nodules on presentation and patients with at least 1 nodule $<10 \mathrm{~mm}$ on presentation $(\mathrm{p}=0.307)$. Of all patients fitting inclusion criteria only, 26 (mean age $=20$, range $7-47,9 \mathrm{~F}, 17 \mathrm{M}$ ) total patients had either a wedge resection, lobectomy, or radiofrequency ablation, with a confirmed 5 year life expectancy of $64 \%(14 / 22)$, and of those 26 patients, 8 had surgeries at any time involving the same lobe as the nodule(s) on presentation and therefore these 8 were excluded from the study. Of these 8 patients, however, only 1 patient had a lung surgery immediately after identifying nodules ( 6 nodules, largest $8 \mathrm{~mm}$ ) on $\mathrm{CT}$ at presentation. Additionally, 2 of these 8 patients had surgeries on the lobe(s) involved with nodules on presentation due to other new nodules and not the original stable nodule. Of the remaining 18 patients who underwent at least 1 lung surgery, 10 of them had nodules on presentation and 8 did not. Of these 10 patient who had nodules on presentation, none had a lung surgery within the first year. 
On presentation, 4 patients had a $1 \mathrm{~mm}$ nodule, 18 patients had a $2 \mathrm{~mm}$ nodule, 11 had a $3 \mathrm{~mm}$ nodule, 5 had a $4 \mathrm{~mm}$ nodule, 3 had a $5 \mathrm{~mm}$ nodule, 2 had a $6 \mathrm{~mm}$ nodule, and 1 had an $8 \mathrm{~mm}$ nodule. On presentation, 24 patients had 1 nodule, 14 patients had 2 nodules, and 6 had $>2$ nodules.

\section{Year Overall Survival and Size of Lung Nodule $<10 \mathrm{~mm}$ on Presentation}

The 5-year OS, calculated using only patients who were confirmed alive or dead at 5 years, for patients with no nodules on presentation was $83 \%$ (34/41), and it was $82 \%(14 / 17), 73 \%(11 / 15)$, and $40 \%(2 / 5)$, for patients in the $1-2 \mathrm{~mm}, 3-4 \mathrm{~mm}$, and $5-9 \mathrm{~mm}$ groups, respectively. A Kaplan-Meier curve comparing 5 year OS of patients with their largest nodules on presentation at each size interval showed there was no difference in 5 year OS in patients with any size nodule $<5 \mathrm{~mm}$ compared to patients with no nodules (figure 2a). Also those who presented with nodules $5-9 \mathrm{~mm}$ had a significantly shorter 5 year OS than those who had no nodules or 1-2 $\mathrm{mm}$ nodules on presentation (figure $2 \mathrm{a}$ ). The mean number of nodules on presentation for the 1-2 mm, 3-4 mm, and 5-9 mm groups were 1.5 ( $\mathrm{SD}=1.1), 2.4(\mathrm{SD}=2.1), 1.7$ (SD $=0.82)$, respectively. ANOVA with Bonferroni multiple comparison indicated that these three groups had similar number of nodules on presentation. There was no difference in percent necrosis, between groups ( $\mathrm{p}$ $=0.91)$.

Additional analysis of additive micronodules size showed that 5 -year OS rates for $0 \mathrm{~mm}$ total, $1-2 \mathrm{~mm}$ total, 3-4 $\mathrm{mm}$ total, and 5-20 mm total were 83\% (34/41), 91\% (10/11), 80\% (8/10), 53\% (8/15), respectively. Kaplan-Meier curves indicated that there was no difference in 5 year OS between any of the groups, however, the $\mathrm{p}$ values when comparing patients with any additive nodule size $5-20 \mathrm{~mm}$ to 0 nodules and 1-2 mm nodules were 0.067 and 0.070 , respectively (figure $2 \mathrm{~b}$ ).

\section{Year Overall Survival and Number of Lung Nodules $<10 \mathrm{~mm}$ on Presentation}

The 5 -year OS for patients with no nodules on presentation was $83 \%(34 / 41)$ and for patients with 1 and [?] 2 nodules had a $90 \%(18 / 20)$ and $53 \%$ (9/17) 5 year OS, respectively. A Kaplan-Meier curve indicates a similar 5 year OS between patients with 0 and 1 nodule on presentation, but patients with 0 and 1 nodules both have a significantly longer 5 year OS than patients with multiple ([?] 2 nodules), (figure 3a). There was no difference in percent necrosis of the patient's primary tumor among these groups $(\mathrm{p}=0.61)$. The mean largest size nodule on presentation for the 1 and 2 nodule groups were $2.7 \mathrm{~mm}(\mathrm{SD}=1.7)$ and $3.2 \mathrm{~mm}$ (SD $=1.2$ ), respectively. A $\mathrm{T}$ test on the mean largest size nodule between these two groups shows no difference in largest size nodule $(\mathrm{p}=0.22)$. However, when only including patient with a largest nodule of [?] $4 \mathrm{~mm}$, there was no significant difference between patients with no nodules and patients with multiple nodules on presentation (figure $3 \mathrm{~b}, \mathrm{p}=0.090$ ). Lastly, when only patents with Huvos scores of 3-4 were included, there was no difference in 5 year OS between patients with no nodules $<10 \mathrm{~mm}$ on presentation and patients with multiple nodules $<10 \mathrm{~mm}$ on presentation (Figure $3 \mathrm{~b}, \mathrm{p}=0.18$ ).

\section{Year Overall Survival and Number of Lobes with Lung Nodules $<10 \mathrm{~mm}$ on Presentation}

Next, the number of lobes involved on presentation was analyzed. The mean size of the largest nodule in patients with one and multiple lobes involved were $2.6 \mathrm{~mm}(\mathrm{SD}=1.6)$ and $3.4 \mathrm{~mm}(\mathrm{SD}=1.2)$, respectively. These two means were not significantly different $(\mathrm{p}=0.10)$. The mean number of nodules in patients with one and multiple lobes involved were $1.1(\mathrm{SD}=0.3)$ and $3.1(\mathrm{SD}=1.9)$, respectively. These two means for number of lobes were significantly different $(\mathrm{p}=0.00049)$. Figure 4 indicates that patients with 2 or more lobes involved have worse 5 year OS than those who presented with 0 or 1 lobe involvement. Of the patients who were confirmed alive or dead at 5 years, 5 year survival rates for 0 nodules, 1 lobe, and multiple lobes were $83 \%(34 / 41), 87 \%(20 / 23)$, and $50 \%$ (7/14), respectively.

\section{Year Overall Survival and Presence of Lung Nodules $<10 \mathrm{~mm}$ within 1 Year of Diagnosis}

The development of nodules within the first year after diagnosis was analyzed. There were 44 patients (mean age $=20,17 \mathrm{~F} 27 \mathrm{M}$ ) who had nodules identified on presentation (defined by nodules detected within the first 2 months), 19 patients (mean age $=21,11 \mathrm{~F}, 8 \mathrm{M}$ ) who developed nodules from 2 months to 1 year after presentation, and 34 patients (mean age $=19,18 \mathrm{~F} 16 \mathrm{M}$ ) who did not develop nodules within the first 
year. Of the patients who developed their first nodules after 2 months, 12 had their first nodules while on chemotherapy and 7 developed their first nodules after finishing chemotherapy. Figure 5a compares 5 year OS between patients who did not have nodules [?] $10 \mathrm{~mm}$ within a year after presentation, patients who had at least 1 nodule [?] $10 \mathrm{~mm}$ within the first 2 months, and patients who did not have nodules in the first 2 months but had at least 1 nodule [?] $10 \mathrm{~mm}$ identified within the first year after diagnosis. Of the patients who were confirmed to be alive or dead at 5 years, the 5 year life expectancies were $96 \%(24 / 25)$, $73 \%(27 / 37)$, and $63 \%(10 / 16)$, respectively. There was no difference between percent necrosis in patients' primary tumor for patients in these three groups $(\mathrm{p}=1.00)$ and there was no relationship between Huvos score and patients in these three groups $(\mathrm{p}>0.73)$. Kaplan Meier curve comparing these three groups indicated that the 5 year OS for patients with no nodules within the first year is significantly different than patients with nodules $<10 \mathrm{~mm}$ on presentation in addition to patients with their first nodules $<10 \mathrm{~mm}$ from 2 months -1 year ( $\mathrm{p}$ values $=0.017,0.0027$, respectively). When only patients with Huvos scores between 3-4 were included, there was no difference in 5 year survival between patients with no nodules $<10$ $\mathrm{mm}$ within the first year of diagnosis, patients with nodules $<10 \mathrm{~mm}$ on presentation, and patients with no nodules on presentation but developed at least 1 nodule between 2 months and 1 year (Figure 5b).

Lastly, the impact demographic categories have on micronodules $<10 \mathrm{~mm}$ on presentation and within the first year were examined on patients who did not undergo pulmonary intervention on the lobe involved (Table 1). Patients 19 and older were more likely to have nodules on presentation than patients 18 and younger. Otherwise, there were no differences found on presence, size, or number of nodules on presentation between all demographic groups investigated.

\section{Discussion}

The 5 -year all-cause survival rate of patients with osteosarcoma has been shown to range from $28 \%$ to $70 \%$. $^{1,2}$ This variation has been attributed to traits such as metastasis at diagnosis, age, tumor location, tumor size, and percent necrosis at primary surgery. However, it has not been well established how the discovery of lung micronodules at the time of diagnosis effects the 5-year overall survival and management. Our retrospective, single institution study of 97 patients demonstrated a $78 \% 5$-year overall survival rate, which is in agreement with historical "localized" OST 5 year survival rates. ${ }^{20,21}$

According to COG guidelines for OST, standard practice has been to remove all visible nodules, however, these recommendations have existed for 25 years, before high resolution CT clearly showed micronodules. In our study the 5 year OS was no different for those who presented with any number of nodules less than 5 $\mathrm{mm}$ in size, without surgical resection on the nodule found on presentation, compared to having no nodules. At presentation, for patients with an additive nodule size less than $5 \mathrm{~mm}$, there was also no difference in 5 -year overall survival in patients without surgical removal of nodules compared to patients with no nodules. Our data suggest surgery (or biopsy) may not be necessary for several small micronodules identified on presentation.

Patients with multiple nodules and lobes on presentation were shown to have a decrease in 5 year OS compared to patients with 0 or 1 nodule or lobe, however, when only patients with nodules $<4 \mathrm{~mm}$ were included there was no difference between patients with multiple nodules and no nodules on presentation. Patients with new micronodules after presentation but before one year had a significantly lower 5-year overall survival (63\%), between that of historical controls for 'localized' and for "metastatic." Additionally, for patients with Huvos scores of 3 or 4 , the 5 year OS for a patient with osteosarcoma did not significantly decrease with number of nodules $<10 \mathrm{~mm}$ on presentation or discovering a nodule $<10 \mathrm{~mm}$ at any time before 1 year, however, these comparisons involved a relatively small number of patients and therefore further investigation are needed to determine the role Huvos scores plays in lung nodule management.

There are a few limitations that need to be addressed. There were generally less patients in the larger size and number of nodule groups, and conclusions would be strengthened if patient numbers particularly in these groups were increased. Additionally, our conformation of patients being alive or dead was dependent on the presence of hospital records indicating patient follow up, which could lead to longer recorded survival 
times for those with continual follow up, however, this should have been relatively similar between groups analyzed.

Other investigators have argued that pulmonary nodules $<5 \mathrm{~mm}$ in OST warrant biopsy/resection because of the 20-30\% incidence of malignancy found on histologic review. ${ }^{17}$ However, just because we can detect OST micro-metastasis, does it mean that it must be removed? The concept of micrometastasis to the lungs has been well established since Link et al, in $1986,{ }^{22}$ long before the availability of high resolution scanning, showed the tremendous benefit of adjuvant chemotherapy in OST patients with "negative" lung CT scans, proving that chemotherapy can and will eradicate sufficiently small lung nodules in most patients that now, and in the future, we are able to "see." As imaging continues to increase sensitivity, understanding the relevance of possibly clinically insignificant subcentimeter nodules is critical to avoid excess treatment and possible morbidity and anxiety for patients. It may be necessary to define an "intermediate group" between "localized" and "metastatic" with an intermediate 5-year survival that has "contaminated" studies on "localized" or "metastatic" OST based on imprecise classification paradigms. This may be based on (1) a solitary nodule less than $<5 \mathrm{~mm}(2)$ uni-lobar involvement (3) a new subcentimer nodule(s) identified on presentation verses after presentation. This group may benefit from different treatment, such as maintenance, and may make it clear if a "low risk" group (no nodules or any number of $1 \mathrm{~mm}$ nodules and nothing developing in the first year) exists that could benefit from less treatment. We hope that prospective COG studies that include observation versus biopsy of micronodules can be performed to help answer these questions.

\section{Conflict of Interest statement}

The authors of this manuscript certify that, to the best of their knowledge, they have no affiliations with or involvement in any organization or entity with any financial interest, have not received and financial support from any pharmaceutical company or other commercial source, or have any non-financial interest (such as personal or professional relationships, affiliations, knowledge or beliefs) in the subject matter or materials discussed in this manuscript.

\section{Acknowledgements}

The authors would like to thank all faculty, nurses, students, hospital staff, and patient family members at Rush University for their efforts in providing care to the patients who were a part of this study.

\section{References}

1. Anderson ME, Randall RL, Springfield DS, Gebhart MC. Chapter 92: Sarcomas of bone. In: Niederhuber JE, Armitage JO, Doroshow JH, Kastan MB, Tepper JE, eds. Abeloff's Clinical Oncology. 5th ed. Philadelphia, Pa: Elsevier; 2014.

2. American Cancer Society. Cancer Facts \& Figures 2020. Atlanta, Ga. American Cancer Society; 2020.

3. Gorlick R, Janeway K, Marina N. Chapter 34: Osteosarcoma. In: Pizzo PA, Poplack DG, eds. Principles and Practice of Pediatric Oncology. 7th ed. Philadelphia Pa: Lippincott Williams \& Wilkins; 2016.

4. Mirabello L, Troisi RJ, Savage SA. Osteosarcoma incidence and survival rates from 1973 to 2004. Cancer. 2009 Apr 1. DOI: 10.1002/cncr.24121.

5. Wang SD, Ren TT, Huang Y, et al. BMPR2 and HIF1-( overexpression in resected osteosarcoma correlates with distant metastasis and patient survival. Chin J Can Res. 2017 Oct;29. DOI: 10.21147/j.issn.1000-9604.2017.05.09.

6. Yao K, Lu MX, et al. Prognostic value of the C-reactive protein to albumin ratio: a novel inflammation-based prognostic indicator in osteosarcoma. Onco Targets Ther. 2017 Nov;2. DOI: 10.2147/OTT.S140560.

7. Picci P, Vanel D, Briccoli A, et al. Computed tomography of pulmonary metastases from osteosarcoma: the less poor technique. A study of 51 patients with histological correlation. Ann Oncol. 2001 Nov;12. DOI: $10.1023 / \mathrm{a}: 1013103511633$.

8. Ginsberg MS, Panicek DM. Subcentimeter pulmonary nodules detected in patients with sarcoma. Sarcoma. 2000. DOI: 10.1080/13577140020008110. 
9. McCarville MB, Lederman HM, Santana VM, et al. Distinguishing benign from malignant pulmonary nodules with helical chest CT in children with malignant solid tumors. Radiology. 2006 May. DOI: 10.1148/radiol.2392050631.

10. Eisenhauer EA, Therasse P, Bogaerts J, et al. New response evaluation criteria in solid tumours: revised RECIST guideline (version 1.1). Eur J Cancer. 2009 Jan. DOI: 10.1016/j.ejca.2008.10.026.

11. Ferrari S, Bacci G, Picci P, et al. Long-term follow-up and postrelapse survival in patients with nonmetastatic osteosarcoma of the extremity treated with neoadjuvant chemotherapy. Ann Oncol. 1997 Aug. DOI: 10.1023/a:1008221713505.

12. Bishop MW, Cheng Y, Krailo MD, et al. Assessing the prognostic significance of histologic response in osteosarcoma: a comparison of outcomes on CCG-782 and INT0133 - a report from the Children's Oncology Group Bone Tumor Committee. Pediatr Blood Cancer. 2016 Oct. DOI: 10.1002/pbc.26034.

13. Petrilli AS, de Camargo B, Filho VO, Bruniera P, Brunetto AL, Jesus-Garcia R. Results of the Brazilian Osteosarcoma Treatment Group Studies III and IV: prognostic factors and impact on survival. J Clin Oncol. 2006 Mar 1. DOI: 10.1200/JCO.2005.03.5352.

14. Pakos EE, Nearchou AD, Grimer RJ, Koumoullis HD, Abudu A, Bramer JA. Prognostic factors and outcomes for osteosarcoma: an international collaboration. Eur J Cancer. 2009 Apr 6. DOI: 10.1016/j.ejca.2009.03.005.

15. Heaton TE, Hammond WJ, Farber BA, et al. A 20-year retrospective analysis of CT-based preoperative identification of pulmonary metastases in patients with osteosarcoma: A single-center review. J Pediatr Surg. 2017 Jan. DOI: 10.1016/j.jpedsurg.2016.10.034.

16. Absalon MJ, McCarville MB, Liu T, et al. Pulmonary nodules discovered during the initial evaluation of pediatric patients with bone and soft-tissue sarcoma. Pediatr Blood Cancer. 2008 Jun. DOI: $10.1002 /$ pbc. 21454 .

17. Kusma J, Young C, Yin H, et al. Pulmonary Nodule Size $<5 \mathrm{~mm}$ Still Warrants Investigation in Patients With Osteosarcoma and Ewing Sarcoma. J Pediatr Hematol Oncol. 2017 Apr. DOI: 10.1097/MPH.0000000000000753.

18. Ghosh KM, Lee LH, Beckingsale TB, et al. Indeterminate nodules in osteosarcoma: what's the followup? Br J Cancer. 2018 Mar 6. DOI: 10.1038/bjc.2017.453.

19. Huvos AG, Rosen G, Marcove RC. Primary osteogenic sarcoma: pathologic aspects in 20 patients after treatment with chemotherapy en bloc resection, and prosthetic bone replacement. Arch Pathol Lab Med. 1977 Jan.

20. Damron TA, Ward WG, Stewart A. Osteosarcoma, chon-drosarcoma, and Ewing's sarcoma. National Cancer DataBase report. Clin Orthop Relat Res . 2007 Jun. DOI: 10.1097/BLO.0b013e318059b8c9.

21. Blackwell JB, Threlfall TJ, McCaul KA. Primary malignantbone tumours in Western Australia, 19721996. Pathology.2005 Aug. DOI: 10.1080/00313020500168737.

22. Link MP, Goorin AM, Miser AW, et al. The effect of adjuvant chemotherapy on relapse-free survival in patients with osteosarcoma of the extremity. N Engl J Med. 1986 Jun 19. doi: 10.1056/NEJM198606193142502.

\section{Legends}

FIGURE 1 Kaplan Meier curve demonstrating survival probability vs years. Groups are separated by presence of a nodule $<10 \mathrm{~mm}$ on presentation and those who did not have any nodules on presentation.

FIGURE 2 Kaplan Meier curves demonstrating survival probability vs years. Groups are separated by a) size of largest nodule $<10 \mathrm{~mm}$ or $\mathrm{b}$ ) sum of nodules $<10 \mathrm{~mm}$ on presentation.

FIGURE 3 Kaplan Meier curves demonstrating survival probability vs years. Groups are separated by a) number of nodules $<10 \mathrm{~mm}$ on presentation b) number of nodules in patients with nodules [?] 4mm, or c) number of nodules in patients with Huvos scores between 3-4

FIGURE 4 Kaplan Meier curves demonstrating survival probability vs years. Groups are separated by number of lobes with lung nodules $<10 \mathrm{~mm}$ on presentation. 
FIGURE 5 Kaplan Meier Curves demonstrating survival probability vs years. Groups are separated by number of nodules $<10 \mathrm{~mm}$ on presentation

TABLE 1 Compares the nodule status of patients based on demographics. $\mathrm{P}$ values generated using t tests and chi squared tests.

\section{Hosted file}

TABLE 1 Compares the nodule status of patients based on demographics.pdf available https://authorea.com/users/364435/articles/484857-pulmonary-micronodules-less-than-5mm-detected-at-presentation-in-patients-with-osteosarcoma-do-not-effect-5-year-overallsurvival
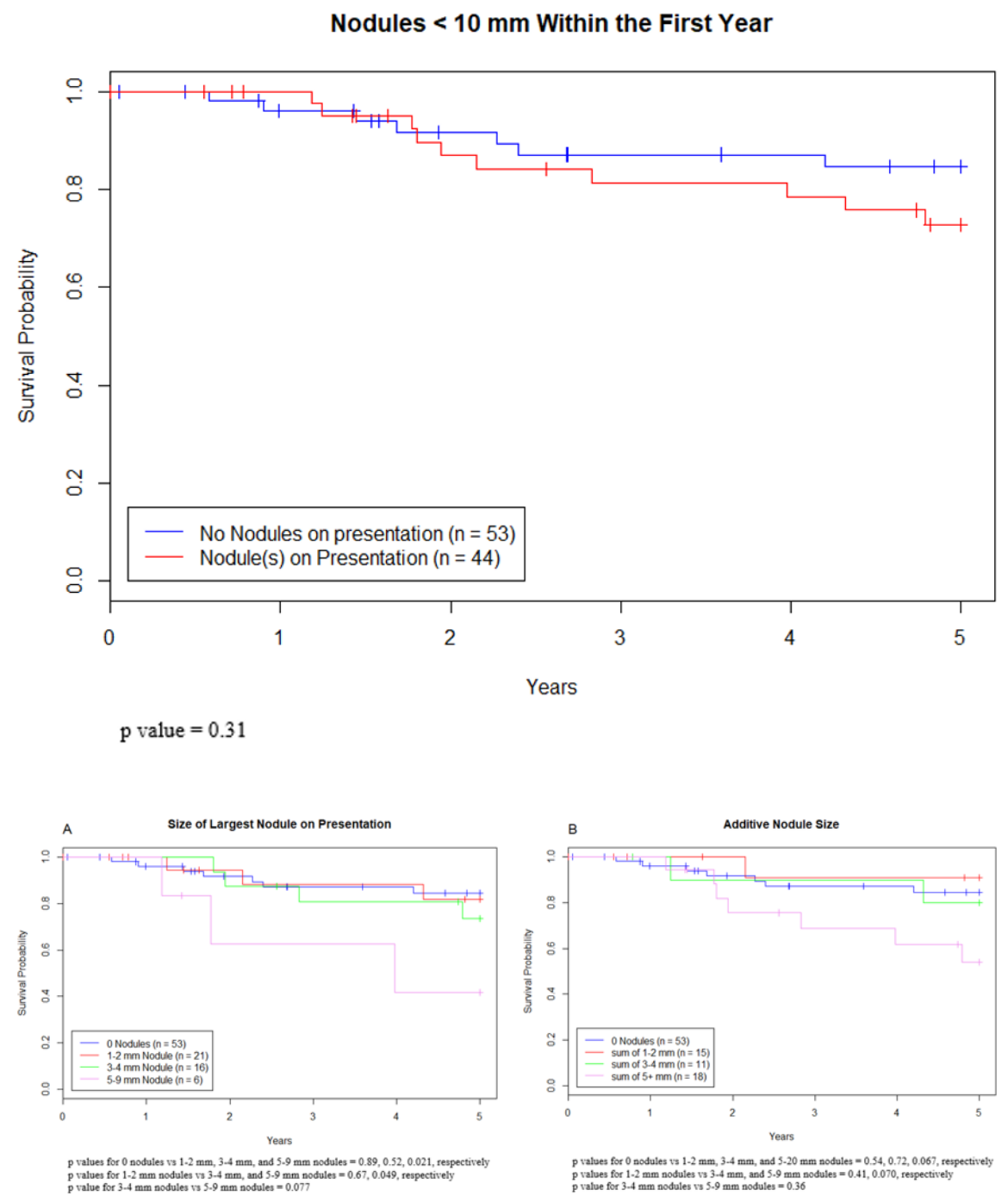

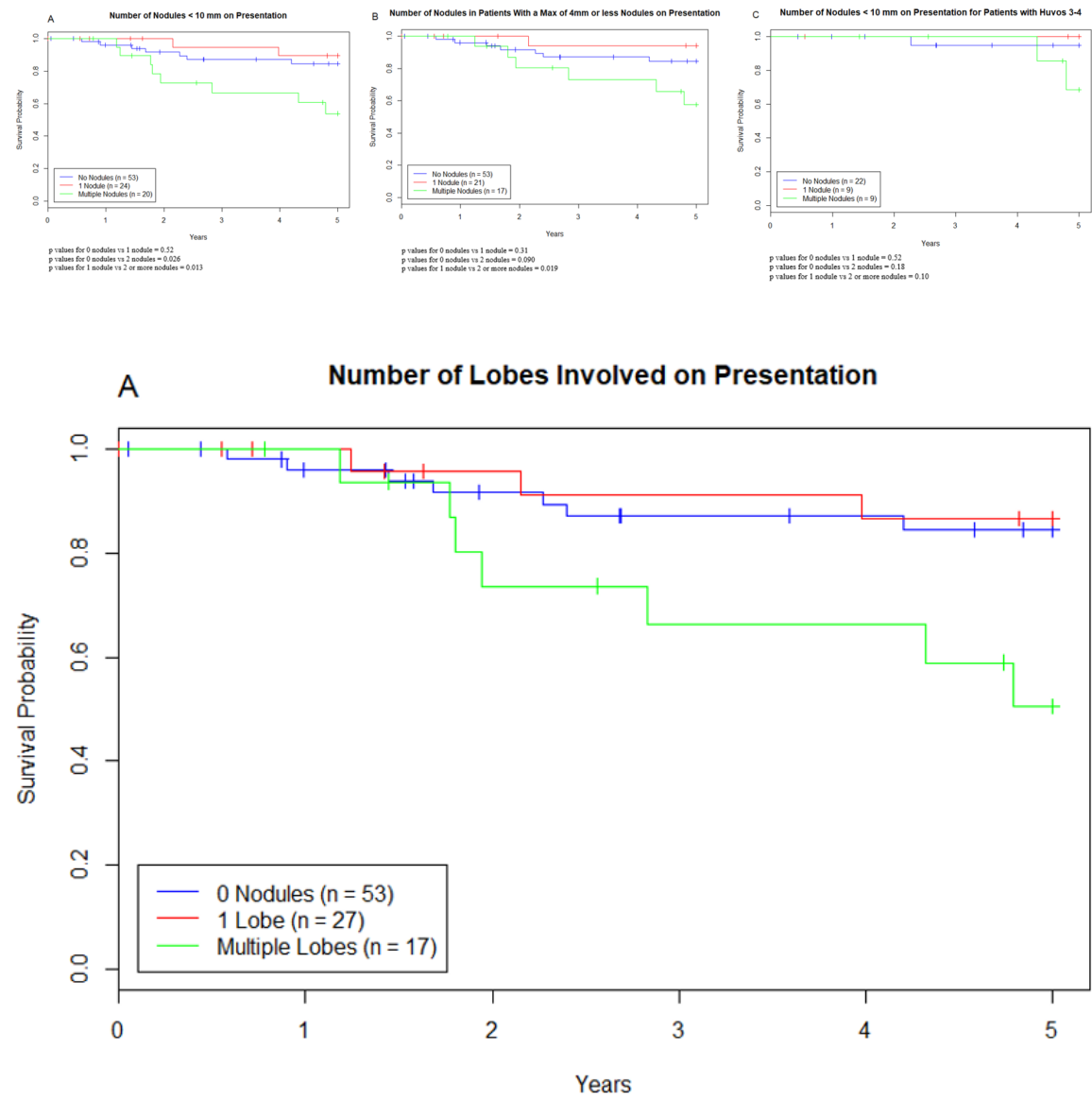

$p$ values for 0 nodules vs 1 lobe and multiple lobes $=0.75,0.034$, respectively $p$ values for 1 lobe vs multiple lobes $=0.025$
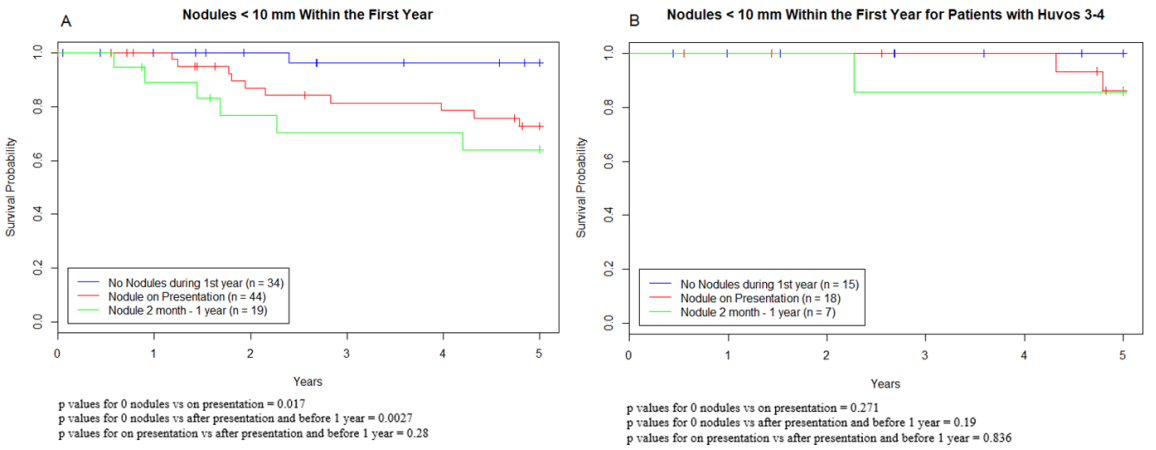